\title{
L'accompagnement : un concept au cœur de l'État social actif. Le cas des pratiques d'accompagnement des personnes handicapées
}

\author{
CHRISTOPHE BARTHOLOMÉ \\ DIDIER VRANCKEN ${ }^{1}$
}

\begin{abstract}
Résumé : L'accompagnement devient un concept clé pour comprendre les nouvelles politiques sociales : désormais, on accompagne les personnes handicapées, les jeunes en difficultés, les chômeurs, les patients, etc. L'intervention sociale se caractérise aujourd'hui par une approche individualisée des suivis et par une recherche constante de l'activation des potentialités et des ressources de l'individu. Cet article s'intéresse aux pratiques d'accompagnement des personnes handicapées en région wallonne, car celles-ci sont, selon nous, une illustration parfaite des nouvelles pratiques sociales relevant de l'État social actif. Nous envisagerons quelques questions essentielles que suscitent ces pratiques innovantes, concernant notamment la contractualisation de l'aide et la responsabilisation des individus mais aussi la conditionnalité de l'aide et la sélection des usagers. Autant de transformations du travail social qui annoncent, selon nous, un nouveau régime de protection à vocation particulariste et incitative.
\end{abstract}

Mots clés : Accompagnement, activation, conditionnalité, contractualisation, handicap, incitation, individualisation, particularisme, potentialité, responsabilisation, ressources, sélection, suivi.

Nées au cours des années 1970, les pratiques d'accompagnement pour personnes handicapées représentent aujourd'hui un terrain tout à fait pertinent pour qui s'intéresse à l'étude des politiques sociales en Belgique. Elles offrent

\footnotetext{
${ }^{1}$ Les auteurs sont professeurs et chercheurs à l'Institut des sciences humaines et sociales, Unité de sociologie de l'organisation et de l'intervention à l'Université de Liège.
} 
une parfaite illustration de l'innovation que représente l'émergence de pratiques de suivis spécifiques et individualisés des usagers de l'aide sociale, qu'il s'agisse de personnes handicapées maintenues dans leurs milieux de vie ou de chômeurs à réinsérer sur le marché de l'emploi. Elles se prêtent également à une lecture des nouvelles politiques d'activation des personnes et des modes d'intervention. Le concept d'accompagnement fait florès dans nombre de secteurs de l'intervention sur les personnes : on accompagne les handicapés, les jeunes en difficultés, les vieux, les mourants, les patients, etc. Désormais, l'accompagnement se décline sans fin dans les méandres des nouvelles politiques sociales.

Sur le plan de l'analyse des politiques et non plus seulement des pratiques, l'étude de l'accompagnement des personnes handicapées permet de saisir l'ampleur d'une distance par rapport à des modes de prise en charge hérités de l'État providence et à un idéal de protection des populations fragilisées. L'accompagnement, tel que pratiqué dans ce secteur, se caractérise par une approche individualisée des suivis et par une recherche constante de l'activation des potentialités et des ressources de la personne. II s'agit désormais, comme nous allons le voir, de rendre les personnes actives plutôt que de se contenter de les prendre en charge ou de les protéger.

Dans cet article, nous verrons combien ces pratiques adressées à un public spécifique apparaissent comme un modèle type des politiques sociales relevant de ce qu'il est aujourd'hui convenu d'appeler l'État social actif. Ainsi, l'accompagnement des personnes handicapées procède-t-il d'une articulation étonnante des conceptions du handicap et des personnes handicapées, de la prise en charge de ces personnes mais aussi du droit, de la subjectivité, de la relation d'aide, des savoirs et des formes d'organisation du travail. Au terme de cette analyse, nous réinterpellerons le concept d'État social actif à la lueur de nos résultats.

\section{Le secteur de l'accompagnement}

\section{A. Un peu d'histoire}

La pratique d'accompagnement naît en Wallonie et à Bruxelles à la fin des années 1970 avec la création d'un petit nombre de services se réclamant de l'accompagnement. Tous ces services émergent dans le paysage social de l'époque grâce à l'initiative de particuliers déjà bien souvent engagés dans le monde du social ou de parents de personnes handicapées. La pratique de l'accompagnement ne relève donc pas au départ d'une volonté particulière des pouvoirs publics de l'époque. L'initiative revient à une poignée d'hommes et de femmes. Le plus souvent, il s'agira de professionnels travaillant déjà dans le secteur du handicap, notamment dans le secteur de l'hébergement, soucieux d'offrir aux personnes handicapées de nouvelles possibilités de prise en charge plus en accord avec leurs demandes et leurs capacités. Cette naissance du secteur de l'accompagnement se fait donc dans un travail de mise à distance, voire d'opposition aux modes de prise en charge des grandes institu- 
tions d'hébergement présentes dans les années 1970 et 1980. Ces grandes institutions, même si elles sont reconnues comme utiles par les praticiens de l'accompagnement, seront généralement critiquées pour leur manque d'écoute de leurs populations et pour leur prise en charge inadéquate en regard des capacités et des potentialités des personnes handicapées. Le modèle asilaire de Goffman (Goffman, 1975) sera souvent mobilisé par les praticiens pour décrire avec force les manquements et les dérives de ces grandes institutions qui enferment derrière des murs.

Au début des années 1980, les services d'accompagnement déjà en place n'étaient encore qu'une poignée et se présentaient comme des initiatives pilotes sans agréments. Ils ne bénéficiaient d'aucune aide financière publique et survivaient grâce au bénévolat. Un processus de conventions annuelles fut mis en place dès 1982 avec le cabinet du ministre chargé des Affaires sociales pour la Communauté française. Cette politique de subsidiation fut effective jusqu'au milieu des années 1990. Les services devaient renégocier avec le cabinet du ministre compétent leurs conventions annuelles. La régulation publique alors en jeu relevait plus d'une politique de subsidiation au coup par coup.

Les années 1980 verront l'apparition de nombreux services d'accompagnement. Le secteur s'étoffera et, dans un même temps, se diversifiera. Pour répondre à cette diversification et aux tensions induites par ce système de subsidiation, certains services émettront le souhait de se regrouper et de se rencontrer. Des formes de regroupement formelles ou informelles s'opéreront progressivement dans le secteur.

\section{B. Le cadre légal}

C'est finalement le 28 juillet 1992 qu'un premier texte de loi relatif aux services d'accompagnement est publié ${ }^{2}$. Les services d'accompagnement soutiennent cette initiative du politique. En effet, ils y voient une possibilité de faire reconnaître leur pratique et d'instaurer une certaine cohérence dans le secteur par la recherche d'un cadre public permettant un subventionnement permanent. Ce décret est le premier texte de loi ayant trait spécifiquement aux services d'accompagnement pour personnes handicapées adultes. Soit une dizaine d'années après que les premières conventions annuelles aient été délivrées. Ce décret donne une première définition d'un service d'accompagnement. II détermine le profil des bénéficiaires: des personnes âgées de 18 ans accomplis, atteintes d'un handicap physique, mental ou sensoriel. Ces personnes doivent vivre hors d'une institution d'hébergement du Fonds de soins médico-socio-pédagogiques pour handicapés ou souhaiter sortir d'une dépendance institutionnelle.

Les accords de la Saint-Quentin de juillet 1993 transféreront un certain nombre de compétences de la Communauté française vers la Région wallonne

\footnotetext{
${ }^{2}$ Décret du 28 juillet 1992 relatif aux services d'accompagnement des personnes handicapées adultes.
} 
parmi lesquelles celles relatives aux services d'accompagnement. Suite à cette redistribution des compétences, l'interlocuteur privilégié des services d'accompagnement sera la Région wallonne et en particulier son ministre des Affaires sociales et de la Santé.

Ainsi, l'arrêté du 13 avril 1995 exécutant le décret du 28 juillet 1992 est-il établi par le gouvernement wallon et non par celui de la Communauté française. Ce décret revêt une importance capitale dans le secteur. En effet, il permet de définir un cadre légal commun aux différents services d'accompagnement. Ce cadre concerne la programmation (un service doit couvrir au moins 50000 habitants), l'identification des bénéficiaires (handicap ou incapacité reconnus), la constitution de l'équipe, l'infrastructure, les normes de fonctionnement (tenue des dossiers, demandes écrites, attestation de reconnaissance du handicap, programme de services ou méthodologie, évaluation), la multidisciplinarité, la concertation, les rapports annuels d'activités, les évaluations et contrôles, les procédures d'agrément, les dépenses, les frais de fonctionnement, la rémunération du personnel.

Le décret du 28 juillet 1992 et l'arrêté d'exécution du 13 avril 1995 constituent donc la base réglementaire des services d'accompagnement. Les premiers agréments commenceront au début de l'année 1997. Cet agrément sera établi pour une période de trois ans et pourra être renouvelé. L'arrêté d'exécution introduit deux éléments pour la régulation des services d'accompagnement : l'instauration d'un cadre administratif commun au secteur et la mise en place d'un nouveau système de financement. Ces deux changements s'expliquent également par l'apparition d'un nouvel acteur dans le champ : l'AWIPH (Agence wallonne pour l'intégration de la personne handicapée). En effet, ce nouvel acteur administratif spécifique (parastatal de type B) sera créé en 1995 sur base des deux anciens dispositifs législatifs ${ }^{3}$ soutenant la politique générale en faveur des personnes handicapées. La création de l'AWIPH sera justifiée par une volonté de mise en cohérence des politiques et de replacer les différentes législations dans un cadre global permettant de nouvelles synergies politiques et une coordination des aides plus efficace. L'AWIPH se verra ainsi confier les missions de deux anciens fonds ainsi que des missions concernant la coordination et l'information mais aussi de gestion et de subventionnement des services agréés.

\section{Situation actuelle}

À l'heure actuelle, les services d'accompagnement de la personne handicapée en Région wallonne sont au nombre de trente-six. Le milieu de l'accom-

\footnotetext{
${ }^{3}$ Ces deux dispositifs législatifs prenaient la forme de deux fonds de financement relevant de domaines d'action spécifiques et distincts. Le Fonds de soins médico-socio-pédagogiques pour handicapés créé en 1967 prenait en charge les frais de logement, d'entretien, de traitement et d'éducation des personnes handicapées mineures et majeures placées en institution. Le Fonds communautaire pour l'intégration sociale et professionnelle des personnes handicapées créé en 1991 sur base de l'ancien Fonds national de reclassement social des handicapés prenait en charge toute la politique concernant l'orientation professionnelle, la formation professionnelle et la mise au travail.
} 
pagnement se caractérise par une grande hétérogénéité. Deux grands types de services d'accompagnement sont identifiables : les services spécifiques et les services généralistes. Les premiers sont spécialisés pour un type de demande bien précis ou pour un profil de bénéficiaire déterminé. Les seconds s'adressent à tous types de handicap (physique, mental, sensoriel) et traitent des demandes variées (logement, travail, formation, gestion budgétaire et administrative, loisirs, etc.). II ne faut toutefois pas conclure à l'homogénéité du travail au sein des services généralistes, la situation y est bien plus complexe. Chaque service, en fonction de son histoire, a tendance à privilégier certains profils de handicap (mentaux légers, physiques, sensoriels) et certaines demandes (mise en autonomie, recherche d'emploi, de loisirs, de logement, guidance administrative ou budgétaire). On constate également que les formations de base des praticiens de l'accompagnement sont très variables et ce, malgré l'instauration d'un cadre-emploi défini par décret pour l'ensemble des services ${ }^{4}$. Cette hétérogénéité se traduit également au niveau des parcours des services, certains sont présents depuis le début des années 1980 et ont contribué au développement du secteur et de la pratique. D'autres ont vu le jour beaucoup plus tardivement et ont donc d'autres sensibilités et modes d'approche du travail.

\section{L'accompagnement en pratique}

\section{A. Une pratique ouverte}

La pratique d'accompagnement est difficilement définissable pour les intervenants de terrain qui n'en précisent pas a priori le contenu. Le concept d'accompagnement renvoie plus à une conception pragmatique (Bechler, 1996), pensée dans l'action, sur base de cas concrets auxquels les praticiens se réfèrent constamment. La méthodologie de l'accompagnement s'est élaborée progressivement au sein des différents services, au gré des expériences de terrain, sans véritable matrice conceptuelle de base. Le contenu méthodologique de la pratique n'a pas été défini dans les textes de loi, laissant le concept totalement ouvert sur la pratique. Ce contexte a permis de garantir aux différents services une grande liberté d'intervention.

Au-delà de cette forte labilité et cette liberté d'intervention, l'accompagnement a pour principal objectif de placer la personne au cœur du processus, de miser sur ses potentialités et ses ressources, mais aussi sur celles de son entourage. II s'appuie sur un substrat à caractère essentiellement relationnel et plus précisément, sur la volonté de recréer du lien social autour de l'usager.

\footnotetext{
${ }^{4} \mathrm{Ce}$ cadre-emploi précise la formation minimum des praticiens : diplôme universitaire ou de niveau A1 pour la fonction de coordination, niveau A1 pour les travailleurs sociaux. Ce cadreemploi prévoit également une base de deux temps pleins et demi par service. On constate également une forte disparité à ce niveau entre les services, certains fonctionnant sur cette simple base, d'autres bénéficient de plusieurs contrats complémentaires (contrats PRIME, PTP, etc.) leur permettant de fonctionner avec une structure beaucoup plus étoffée.
} 
Comme nous l'avons déjà précisé plus haut, les services d'accompagnement ont construit leurs pratiques en réaction aux modes de prise en charge en vigueur dans les institutions fermées. Le secteur de l'accompagnement s'est donc progressivement construit et structuré parallèlement (voire en opposition) à un imaginaire réadaptatif et rééducatif (Stiker, 1984) caractéristique des politiques du handicap à cette époque et très fortement orienté vers la réinsertion à l'emploi (Ebersold, 1992).

Nous avons pu identifier au sein du secteur trois principes fondamentaux communs à l'ensemble des pratiques du secteur : le travail à la demande, le travail au cas par cas et «faire avec » la personne handicapée au lieu de " faire pour ". Ces trois principes nous semblent se distinguer plus par leur dimension relationnelle que par leur véritable substance. Ils semblent davantage proposer un cadre global à la relation d'accompagnement qu'un contenu précis dictant le travail concret à réaliser. Les intervenants se retrouvent tous sur la nécessité d'appréhender le bénéficiaire en tant que personne et non plus en tant qu'objet d'une catégorisation. Ils insistent sur la nécessité de partir d'une demande exprimée par l'usager et qui pourra ensuite déboucher sur la construction d'un projet. Cette expression d'une demande peut prendre des formes très diverses mais doit témoigner d'une envie, d'une volonté de la part de la personne handicapée de changer quelque chose dans sa vie. Cette notion de demande est souvent érigée par les praticiens comme une condition nécessaire pour la mise en place d'un accompagnement, et ce pour deux raisons. La première raison relève d'un principe déontologique. Les praticiens de l'accompagnement refusent d'entrer dans une démarche d'" aidecontrainte ", situation où la personne handicapée se voit aidée sans qu'elle en ait réellement exprimé le souhait. L'intervention du service d'accompagnement n'est donc légitime que si elle est sollicitée véritablement par la personne handicapée elle-même. La deuxième raison est d'ordre pratique. Un accompagnement à la demande est synonyme pour les praticiens d'un accompagnement de qualité. En effet, sur base de leur expérience, les praticiens savent qu'un accompagnement valable et pertinent ne peut être réalisé sans une participation et une implication réelles de la personne handicapée. Au-delà de l'expression d'une demande, se révèle donc une normativité nouvelle adressée aux personnes handicapées, exhortées à s'impliquer, à s'engager dans le processus d'aide qu'elles sollicitent. Cette expérience explique également cette volonté farouche des praticiens de définir la personne accompagnée comme l'acteur principal de son projet et de son accompagnement. En découle le second principe : « faire avec » la personne et non « faire pour » ou " faire à la place de ". II est exclu que la personne handicapée se présente comme un ayant-droit ou comme un simple client réclamant un service. La formule consacrée des praticiens pour traduire cette idée est éloquente : " nous ne sommes pas un service taxi ou un service bancaire ». Les pratiques d'accompagnement sont donc orientées vers un idéal actif. Un effort particulier est exigé de la part de la personne handicapée afin qu'elle se prenne progressivement en main, mobilise et exploite ses compétences, développe et utilise tout un réseau relationnel autour d'elle. Dans cette perspective, l'approche au cas par cas est également érigée en principe méthodologique fondamental. Travailler sur base des compétences, des potentialités, des ressources et du 
réseau relationnel de la personne, entraîne une forte individualisation et particularisation de l'aide apportée à la personne. Cette approche au cas par cas s'explique également par la volonté manifeste des praticiens de travailler sur base des situations réelles vécues par les personnes qui ne peuvent être appréhendées et traitées selon une approche trop fortement standardisée et formalisée. Les praticiens tiennent particulièrement à la prise en compte du parcours de vie de la personne et de ses attentes, au respect de son style de vie et de ses choix.

\section{B. Contractualisation de l'aide et responsabilisation de l'usager}

L'accompagnement appréhende l'individu dans une grande diversité de registres, ayant abandonné le carcan trop étroit de la catégorie " handicapé ». Cet individu est désormais une personne ayant un handicap ${ }^{5}$, ayant sa place dans la société et le droit de définir sa trajectoire personnelle, d'être acteur de son projet de vie. Par moments, cette personne sera qualifiée par les praticiens de "bénéficiaire ", à d'autres moments elle se verra définie comme " usager ", supposant la reconnaissance de son altérité relationnelle mais aussi juridique (Chauvière, 2004). La personne handicapée se voit ainsi reconnue comme un citoyen à part entière ayant des droits mais aussi des devoirs. II n'est dès lors pas étonnant de constater que tout accompagnement repose sur l'établissement d'un contrat passé entre la personne handicapée et le service. $\mathrm{Ce}$ contrat portera sur les engagements et les droits de chacune des parties. L'utilisation du contrat démontre la volonté des praticiens de l'accompagnement de sortir d'une logique de dépendance et d'assistanat des individus en les faisant participer de manière plus active. Car le contrat d'accompagnement est moins un contrat au sens strict du terme qu'un outil de travail, permettant de donner du sens à la relation et de clarifier la situation d'aide (Cousin, 1996). Depuis le début des années 1990, l'utilisation du contrat se développe dans les pratiques d'aide sociale. Cette utilisation a parfois été dénoncée car l'instauration du contrat implique un certain nombre d'exigences, à savoir l'engagement de la personne dans un processus d'aide et son adhésion à un projet. Ce ne sont pas ces exigences en tant que telles qui posent problème mais bien davantage le fait que le travailleur social sera bien souvent le seul évaluateur de cet engagement de la personne. Dès lors, la dérive méritocratique n'est pas loin, teintée de surcroît d'une subjectivité ressemblant très fort à de l'arbitraire. Cette évaluation subjective du comportement de la personne handicapée se retrouve bien dans la pratique d'accompagnement. Ainsi, les praticiens relatent des situations d'accompagnements abandonnés car les personnes suivies ne montraient pas suffisamment d'implication ou d'engagement. On constate globalement une tendance de fond à une responsabilisation accrue de l'usager. En effet, la réussite d'un accompagnement est aujourd'hui très clairement liée aux bonnes dispositions de la personne à s'impliquer, à participer et à s'engager. Si ce phénomène d'élargissement de la responsabilité est constaté notamment auprès d'autres publics et notamment des populations de

${ }^{5}$ Ou plus précisément, vivant une situation de handicap. 
chômeurs (Franssen, 2003), ce phénomène est tout aussi interpellant pour les populations de personnes handicapées car cette responsabilisation se fait parfois en ignorant totalement les déficiences objectives et bien réelles de ces populations (Ebersold, 2003). Cet élargissement de la responsabilité de l'usager rend la question du dénouement du processus d'accompagnement très délicate pour les praticiens. Peut-on parler d'échec pour une personne dont le projet d'autonomie ne s'est pas avéré concluant, personne qui décide de retourner dans une institution d'hébergement? Ce type de raisonnement est difficilement tenable pour les praticiens qui préféreront souligner le caractère positif de l'expérience et le risque d'avoir osé entreprendre et expérimenter autre chose.

\section{Conditionnalité de l'aide et sélection des publics}

Dans la foulée, les praticiens de l'accompagnement précisent généralement que l'accompagnement ne convient pas et n'est pas destiné à toutes les personnes handicapées. Les anciennes formes de prise en charge (hébergement notamment) ont toujours leur raison d'être et sont considérées comme complémentaires à l'approche spécifique de l'accompagnement. On peut en conclure que les praticiens de l'accompagnement ont progressivement mis en place un principe de conditionnalité de l'aide. Ces conditions portent sur l'engagement et l'implication de la personne dans le processus d'aide et dans son projet. Sur base de ces exigences comportementales, les praticiens de l'accompagnement effectuent un travail de sélection des usagers, réorientant certains vers d'autres dispositifs d'aide considérés comme plus adaptés au profil ou à la nature de la demande. Cette sélection et cette réorientation des individus sont toujours motivées par un souci d'efficacité qui anime les praticiens de l'accompagnement. Cependant, il est intéressant de constater qu'une personne handicapée ayant obtenu de l'administration une autorisation d'accompagnement pourra se voir refusée et réorientée par le service d'accompagnement qu'elle sollicitera. Cette sélection et cette réorientation se feront pour des raisons diverses allant de la nature de la demande inadaptée à la pratique d'accompagnement, du manque d'implication de la personne ou tout simplement pour cause de surcharge de travail momentanée du service (et donc une incapacité pour le service d'offrir un accompagnement de qualité). Mais malgré ces raisons tout à fait louables, on ne peut s'empêcher un questionnement sur la part d'arbitraire qui caractérise ce travail de sélection. Les professionnels de l'accompagnement reconnaissent qu'en fonction de la conjoncture spécifique du service, d'une compatibilité relationnelle, d'une situation spécifique interpellant davantage le travailleur social, ces critères de sélection seront aménagés. Derrière ces aménagements, se pose la question de l'égalité d'accès des individus à ce type de dispositifs d'aide aux personnes. Certes, on peut montrer que la sélection permet d'apporter aux personnes une aide de qualité et réellement pertinente. Toutefois, le principe de conditionnalité et le travail de sélection nous apparaissent caractéristiques de ces mécanismes de trappe, de filtrage des publics cibles qui s'opèrent au sein de l'État social actif, aboutissant parfois à la construction de véritables nasses 
pour les publics les plus précarisés (Vrancken, 2002). La nature quelque peu arbitraire de ce travail de sélection opéré au coup par coup et le manque de réflexion politique quant à ses conséquences concrètes pour les populations (les travailleurs sociaux prenant en charge la responsabilité de cette sélection et non les organismes de tutelle) ont à terme des effets d'entraînement considérables sur les modes mêmes de réorganisation de nos politiques sociales. Le malaise n'est pas loin quand on s'interroge sur le devenir de ces individus refusés. II y a là toute une réflexion globale à mener sur le sens induit par de nouveaux dispositifs visant à travailler au plus près des personnes et de leurs attentes.

\section{Conclusions : un État social actif... en action !}

II règne aujourd'hui, dans le milieu des analystes belges des politiques sociales, un consensus pour reconnaître que l'État social actif a été importé en Belgique à la fin des années 1990, apparaissant clairement dans la déclaration gouvernementale de la majorité «arc-en-ciel » de 1999. C'est même devenu, pourrait-on ajouter, un lieu commun. La découverte et l'utilisation par nos hommes politiques de cette notion suscitent souvent la méfiance. L'évocation du vocable d'État social actif est souvent qualifiée de discours idéologique par certains chercheurs, de roue de secours à la crise des États providence occidentaux. Et on ne peut fondamentalement leur donner tort.

Notre analyse des pratiques d'accompagnement des personnes handicapées a pu mettre en évidence l'ampleur d'une évolution toutefois amorcée bien en amont au niveau des pratiques de terrain. Nous trouvons ainsi trace de cette évolution dès la fin des années 1970 et début des années 1980. La pratique d'accompagnement n'a cessé d'évoluer mais on peut affirmer qu'elle est entrée en résonance avec les nouvelles attentes des pouvoirs publics au cours des années 1990. Qu'en tirer comme conclusions?

Fondamentalement, nous vivons aujourd'hui une phase de déplacement de nos politiques sociales, impliquant le remplacement d'un système d'octroi de droits objectifs par une série de dispositions individualisantes ancrées sur les comportements des acteurs, visant à les responsabiliser davantage. Chaque citoyen se verrait ainsi responsable de sa santé, responsable de sa retraite, responsable de sa réinsertion sur le marché du travail. En travaillant à la marge des États sociaux, on aurait permis à leur structure de perdurer en insufflant les modifications les plus conséquentes dans les dispositifs les moins universalistes. Ainsi, les évolutions les plus notoires de nos politiques sociales ont-elles été opérées, au cours de ces dernières années, à la marge des systèmes sociaux, à partir de droits conditionnels en direction de populations ciblées et localisées sur le territoire. Comme l'observe Castel (2003), ces évolutions n'ont pas répondu à une politique d'ensemble, tel en témoigne notre cas d'étude qui s'est développé dans le confinement de pratiques spécifiques pour les personnes handicapées. Mais à force de petits pas, ces évolutions ont contribué à un « nouveau régime de protection » différent du précédent, alors caractérisé par l'octroi de protections fondées sur le travail. Cette évolution actuelle implique un changement radical de perspective par un recentrage des 
politiques sur les personnes placées en dehors du régime commun. Au régime universaliste et égalitaire précédent, viendrait s'adjoindre un nouveau régime à vocation particulariste et incitative. Ce nouveau régime s'appuierait progressivement sur des mesures de discriminations positives, des prestations ciblées (et non uniformes) à partir de besoins sociaux spécifiques, de formules d'interventions décentralisées, décloisonnées, transversales, misant sur le partenariat, la mobilisation des réseaux propres des bénéficiaires et de ceux des intervenants professionnels.

\author{
Christophe BARTHOLOMÉ \\ Institut des Sciences humaines et sociales \\ Bat. B31 - Sociologie de l'organisation \\ et de l'intervention sociale \\ Bd du Rectorat, 7 \\ B 4000 Liège 1 \\ Tél. : 0032.4.366.27.42 \\ Fax : 0032.4.366.29.83 \\ 0032.4.366.47.51
}

Courriel : christophe.bartholome@ulg.ac.be

\author{
Didier VRANCKEN \\ Institut des Sciences humaines et sociales \\ Bat. B31 - Sociologie de l'organisation \\ et à l'intervention \\ $B d$ du Rectorat \\ B 4000 Liège 1 \\ Tél. : 0032.4.366.31.76 \\ Courriel : didier.vrancken@ulg.ac.be
}

\title{
Bibliographie
}

BAELDE P., COPPIN B., LE CERF J.-F., MOUREAU B., Comprendre et accompagner, les parents avec une déficience intellectuelle, Paris, Gaëtan Morin, 1999.

BECHLER Pierre, «Accompagnement des personnes adultes handicapées mentales : du concept à la formation des professionnels ", Revue Européenne du Handicap Mental, vol. 3, no 12, p. 19-32.

CASTEL Robert, L'insécurité sociale. Qu'est-ce qu'être protégé ?, Paris, Le Seuil, 2003.

CHAUVIĖRE Michel, Le travail dans l'action publique. Sociologie d'une qualification controversée, Paris, Dunod, 2004.

CHAUVIÈRE Michel, GODBOUT Jacques T. (Éds), Les usagers entre marché et citoyenneté, Paris, L'Harmattan, 1992.

COUSIN Olivier, « Les mutations du travail social : de la transformation du public aux changements dans les modes de prise en charge ", Sociologie du travail, $n^{\circ} 2,1996$, p. 141-161. EBERSOLD Serge, L'invention du handicap. La normalisation de l'infirme, Paris, CTNERHI, 1992.

EBERSOLD Serge, "Gestion individualisée des risques sociaux et crise des catégories instituées ", Éducation permanente, vol. 3, n 156, 2003, p. 13-25.

FRANSSEN Abraham, “Les nouvelles figures de la question sociale ", La Revue Nouvelle, Bruxelles, décembre 2003, p. 10-61. 
GOFFMAN Erving, Asiles. Études sur la condition sociale des malades mentaux, Paris, Éd. Minuit, coll. "Le Sens Commun », 1975.

STIKER Henri-Jacques, Corps infirmes et sociétés, Paris, Aubier, 1984.

VRANCKEN Didier, Le crépuscule du social, Bruxelles, Labor, coll. « Quartier Libre », 2002. VRANCKEN Didier, BARTHOLOMÉ Christophe, RENOUPREZ Christophe, Les pratiques d'accompagnement pour personnes handicapées en question? Vers un nouveau modèle d'action publique ? Université de Liège, Rapport de recherche à l'Association des Services et d'Accompagnement pour Handicapés dans le cadre d'une subvention accordée par le ministre des Affaires sociales et de la Santé T. Detienne, 2001. 